\title{
Use of a negative selectable marker for rapid selection of recombinant vaccinia virus
}

\author{
Stacy D. White ${ }^{1,2, *}$, Kip Conwell ${ }^{1,2, *}$, Jeffrey O. Langland ${ }^{1,4}$, and Bertram L. Jacobs ${ }^{1,2,3}$ \\ ${ }^{1}$ Center for Infectious Diseases and Vaccinology, Biodesign Institute, Arizona State University, Tempe, Arizona, USA \\ ${ }^{2}$ Graduate Program in Molecular and Cellular Program, Arizona State University, Tempe, Arizona, USA, ${ }^{3}$ Faculty \\ of Biomedicine and Biotechnology, School of Life Science, Arizona State University, Tempe, Arizona, USA, and \\ ${ }^{4}$ Department of Naturopathic Research, Southwest College of Naturopathic Medicine and Health Sciences, Tempe, \\ Arizona, USA
}

BioTechniques 50:303-309 (May 2011) doi 10.2144/000113667

Keywords: Recombinant; coumermycin; gyrase-PKR; Vaccinia virus; E3L; selection

${ }^{*}$ S.D.W. and K.C. contributed equally to this work.

Vaccinia virus has been a powerful tool in molecular biology and vaccine development. The relative ease of inserting and expressing foreign genes combined with its broad host range has made it an attractive antigen delivery system against many heterologous diseases. Many different approaches have been developed to isolate recombinant vaccinia virus generated from homologous recombination; however, most are time-consuming, often requiring a series of passages or specific cell lines. Herein we introduce a rapid method for isolating recombinants using the antibiotic coumermycin and the interferon-associated PKR pathway to select for vaccinia virus recombinants. This method uses a negative selection marker in the form of a fusion protein, GyrB-PKR, consisting of the coumermycin dimerization domain of Escherichia coli gyrase subunit $B$ fused to the catalytic domain of human PKR. Coumermycin-dependent dimerization of this protein results in activation of PKR and the phosphorylation of translation initiation factor, eIF $2 \alpha$. Phosphorylation of this factor leads to an inhibition of protein synthesis, and an inhibition of virus replication. In the presence of coumermycin, recombinants are isolated due to the loss of this coumermycin-sensitive gene by homologous recombination. We demonstrate that this method of selection is highly efficient and requires limited rounds of enrichment to isolate recombinant virus.

Vaccinia virus (VACV), the poxvirus used as the vaccine against smallpox, has gained widespread use as a general vector for expressing foreign proteins in mammalian cells. The ability to take up large inserts of DNA and express high levels of foreign protein in a wide variety of cell lines has made VACV an attractive delivery vehicle for expressing antigens and analyzing protein function (1). The standard method of generating VACV recombinants is through homologous recombination between replicating viral DNA and a transfected plasmid or PCR product containing the protein coding sequence of interest flanked by viral sequences (1). The frequency of recombination accounts for $\sim 0.1 \%$ of total virus, and isolation of the recombinants typically requires a series of selections using a genetic marker $(1,2)$.

Many different selection schemes have been developed for detection of VACV recombinants. Such examples include the use of thymidine kinase-negative and -positive selection $(3,4)$, reversal of host range mutations $(5-7)$, neomycin resistance (8), and transient dominant selections using mycophenolic acid (MPA)(1). These methods have proven to be quite efficient, but they often require special cell lines or serial passages in selection media and therefore can be time-consuming.

Herein we demonstrate a novel, alternative method for rapid isolation of recombinant VACV using the antibiotic coumermycin and a coumermycin-sensitive VACV, VACVAE3L::GyrB-PKR. The VACVAE3L::GyrB-PKR expresses the coumermycin dimerization domain of Escherichia coli gyrase B (GyrB, residues 1-220) fused to the catalytic domain of mammalian dsRNA-dependent protein kinase, PKR (residues 258-551), in the viral locus of interest for gene insertion. The GyrB-PKR system has been characterized previously (9). In this heterologous system, the gyrase domain binds to coumer- mycin in a 2:1 stoichiometric ratio, thereby causing the dimerization of the PKR kinase domain (Figure 1A) (10). This dimerization mimics the endogenous activation of PKR and results in the phosphorylation of translation initiation factor eIF $2 \alpha$, which leads to an inhibition of protein synthesis $(11,12)$. For this study, we used the E3L locus of VACV to express GyrB-PKR. In this system, recombinants are generated by standard homologous recombination between a plasmid containing the insert of interest surrounded by sequences that flank the E3L gene and the parental VACVAE3L::GyrB-PKR. Recombinants are resistant to the effects of coumermycin, whereas the replication of VACVAE3L::GyrB-PKR is inhibited following treatment with coumermycin. Therefore, this system exclusively allows the viability of viruses that undergo a double recombination event that result in loss of the GyrB-PKR gene and retention of the desired insert. 


\section{Materials and methods}

Cells, virus and reagents

Baby hamster kidney (BHK-21-CCL10) and rabbit kidney cells (RK13-CCL37) (ATCC, Manassas, VA, USA) used in the experiments were maintained in MEM (Mediatech, Manassas, VA, USA) supplemented with 5\% FBS (HyClone, Logan, UT, USA) and $10 \mu \mathrm{g} / \mathrm{mL}$ gentamicin (Invitrogen, Carlsbad, CA, USA) at $37^{\circ} \mathrm{C}$ with $5 \%$ $\mathrm{CO}$. Coumermycin A1 (Sigma-Aldrich, St. Louis, MO, USA) was dissolved in DMSO (Invitrogen) at a stock concentration of $5 \mathrm{mg} / \mathrm{mL}$ and diluted with PBS. Vectors pC939 and pC940 containing GyrB-PKR and GyrB-PKRK296H, respectively, were provided by Tom Dever (National Institutes of Health, Bethesda, MD, USA) and have been described (9). These vectors contain the residues 1-220 of E.coli gyrase B fused to the kinase domain (residues 258-551) of human PKR. The catalytic inactive mutant contains a lysine to histidine mutation at residue 296 of the PKR domain. Viruses used in this study expressed the GyrB-PKR or its mutant protein in either the E3L or the $\mathrm{J} 2 \mathrm{R}$ locus. Isolation of VACV expressing GyrB-PKR was by transient dominant selection using mycophenolic acid as describe previously $(13,14)$. Individual mycophenolic acid-resistant plaques were tested for sensitivity to coumermycin, and the most coumermycin-sensitive viruses were further characterized. VACV strains used were Western Reserve (WR) and NYVAC. All infections, plaque purifications, virus amplifications and viral genomic extraction for sequencing were carried out as previously described (14).

Coumermycin sensitivity of

VACVDE3L, VACVDE3L::GyrB-PKR, VACVAE3L::GyrB-PKRK296H, and VACVAJ2R::GyrB-PKR

RK13 cells grown in six-well dishes were treated with coumermycin A1 at doses ranging $0-100 \mathrm{ng} / \mathrm{mL}$ in media for $16 \mathrm{~h}$. The cells were infected with $100 \mathrm{pfu}$ VACVAE3L, VACVAE3L::GyrB-PKR, or VACVAE3L::GyrB-PKRK296H, and the infections were carried out in media supplemented with the corresponding concentration of coumermycin for $48 \mathrm{~h}$. To test the sensitivity of the VACVAJ2R::GyrB-PKR to coumermycin, RK13 cells were mocktreated or treated with coumermycin $\mathrm{A} 1$ at a concentration of $100 \mathrm{ng} / \mathrm{mL}$ for $24 \mathrm{~h}$ and then infected with 10 -fold serial dilutions of the virus stock. Infections were carried out for $48 \mathrm{~h}$ in media with or without $100 \mathrm{ng} / \mathrm{mL}$ of coumermycin A1. Cells were stained with crystal violet and plaques were analyzed by a standard plaque assay.

A

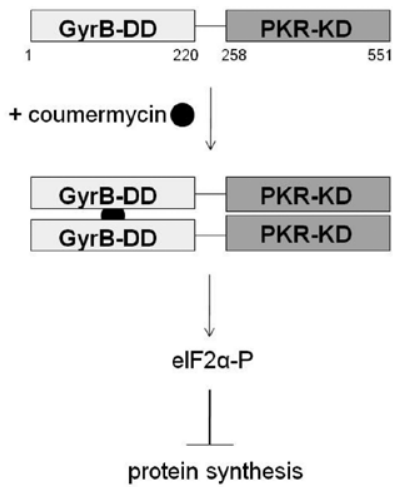

Figure 1. Coumermycin/GyrB-PKR system as a negative selection marker for isolation of VACV recombinants. (A) Schematic representation of the coumermycin/PKR system. Coumermycin-induced dimerization of GyrB-PKR fusion protein results in activation of the fused PKR catalytic domain. The GyrB-PKR fusion protein consists of the first 220 amino acids of the $E$. coli gyrase B coumermycin-dependent dimerization domain (GyrB-DD) fused to residues 258-551 of the catalytic domain of human PKR (PKR-KD). The GyrB domain binds to coumermycin in a 2:1 ratio, resulting in the dimerization and activation of the kinase domain of PKR. This leads to the phosphorylation of translation initiation factor elF2a, causing an inhibition of protein synthesis. (B) Expression of GyrB-PKR and GyrB-PKRK296H in recombinant VACV. RK13 cells were infected at an $\mathrm{MOI}$ of 5 with the indicated VACV mutants. At $6 \mathrm{~h}$ post-infection, cell extracts were harvested and subjected to Western blot analysis using antibodies that recognize human PKR.

Western blot analysis for GyrB-PKR and eIF2a phosphorylation

For GyrB-PKR expression analysis, subconfluent RK13 cells were mock-treated or treated with $100 \mathrm{ng} / \mathrm{mL}$ coumermycin A1. After $16 \mathrm{~h}$, the cells were infected with VACVAE3L, VACVAE3L::GyrB-PKR or VACVAE3L::GyrB-PKRK296H at a multiplicity of infection (MOI) of $5 \mathrm{pfu} / \mathrm{cell}$. At $6 \mathrm{~h}$ post-infection, the cells were scraped into media and pelleted by centrifugation at $500 \times g$ for $10 \mathrm{~min}$ at $4^{\circ} \mathrm{C}$. The cells were lysed by addition of RIPA lysis buffer ( $1 \times$ PBS, $0.1 \%$ SDS, $1 \%$ nonyl phenoxypolyethoxylethanol (NP40), $0.5 \%$ sodium deoxycholate, and $100 \mathrm{mM}$ sodium fluoride) followed by incubation on ice for $10 \mathrm{~min}$. The lysates were centrifuged at $10,000 \times g$ for $10 \mathrm{~min}$ at $4^{\circ} \mathrm{C}$ and the supernatant transferred to new tube. Equal amounts of $2 \times$ SDS loading buffer was added to the lysates and then separated on a $12 \%$ SDS PAGE gel under denaturing conditions and then transferred onto PVDF membrane. Following transfer, the membranes incubated in blocking buffer (3\% nonfat dry milk, $140 \mathrm{mM} \mathrm{NaCl}, 3 \mathrm{mM}$ $\mathrm{KCl}, 20 \mathrm{mM}$ Tris pH 7.8, 0.05\% Tween 20) for $1 \mathrm{~h}$ at room temperature. GyrB-PKR expression was determined by using rabbit antibodies directed against total PKR and the phosphorylation of eIF $2 \alpha$ by phosphospecific eIF $2 \alpha$ antibodies (Cell Signaling, Danvers, MA, USA) at a concentration of 1:1000 diluted in blocking buffer. Secondary goat anti-rabbit antibodies conjugated to HRP (Sigma-Aldrich) were added at 1:15,000 in blocking buffer followed by chemiluminescent development (Supersignal West Dura, Thermo Scientific, Rockford, IL, USA).

Generation of VACV $\Delta E 3 \mathrm{~L}: \mathrm{LacZ}$

by coumermycin system

In vivo recombination (IVR) of the following viruses occurred accordingly: subconfluent $\mathrm{BHK}$ cells $\left(2 \times 10^{6}\right.$ cells total) were transfected with $2 \mu \mathrm{g}$ pMPLacZ using Lipofectamine and Plus Reagent (Invitrogen) per the manufacturer's directions and coinfected with parental VACVAE3L::GyrB-PKR at an MOI of $0.05 \mathrm{pfu} / \mathrm{cell}$. At $30 \mathrm{~h}$ post-infection, cells were scraped and virus was released by three rounds of freeze-thaw treatment followed by sonication. RK13 cells pretreated with or without coumermycin A 1 at $100 \mathrm{ng} / \mathrm{mL}$ for $16 \mathrm{~h}$ were infected with dilutions of the IVR extract, and overlaid with media containing coumermycin if required. After $48 \mathrm{~h}$ post-infection, the cells were covered with an agarose overlay consisting of $1 \times \mathrm{MEM}, 1.5 \%$ agarose, $\mathrm{X}$-gal substrate $(10 \mathrm{mg} / \mathrm{mL})$, and neutral red. At 24 h postinfection, recombinant plaques (blue) were quantified and compared with the number of parental plaques (clear).

\section{Results and discussion}

Parental virus (VACVAE3L::GyrB-PKR) was constructed to express the first 220 amino acids of E.coli GyrB fused to residues $258-551$ of the kinase domain of human PKR in the E3L locus of VACV. As described in the "Materials and methods" section, 


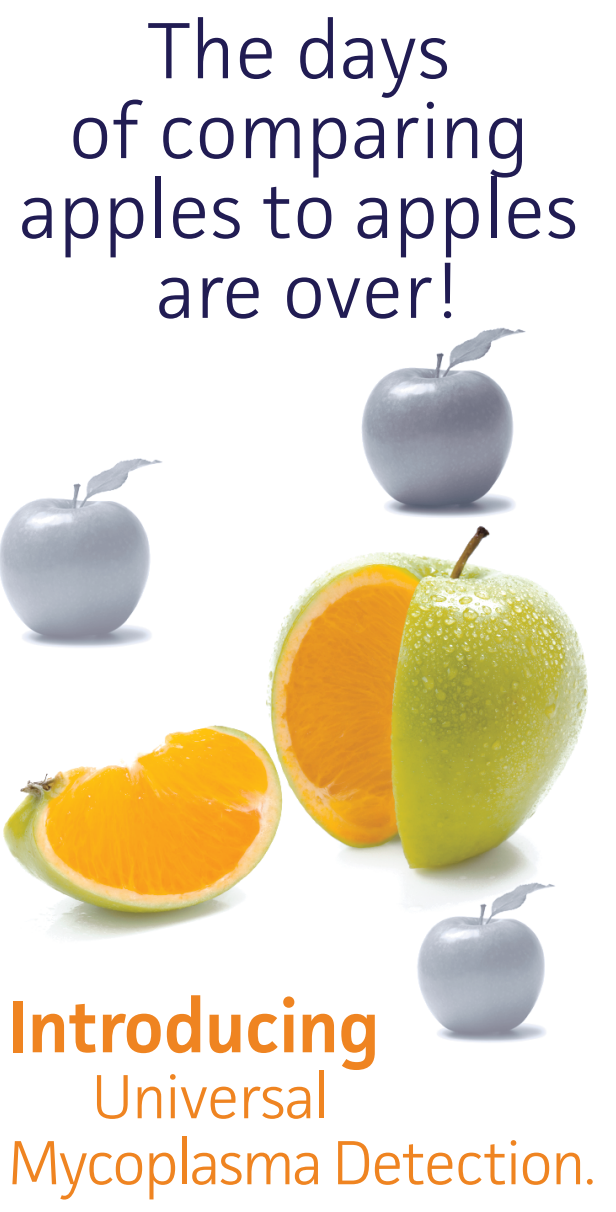

\section{Not all PCR-based kits are the same.}

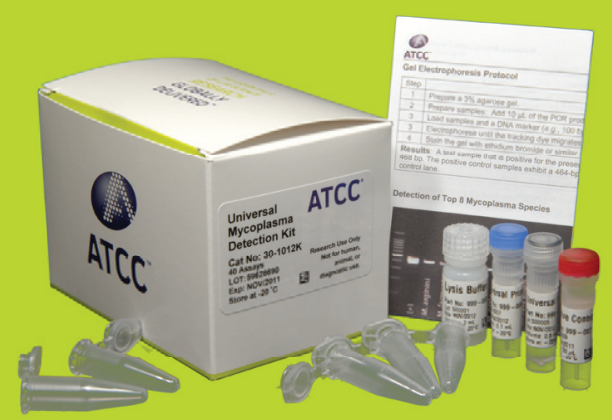

- Superior sensitivity

- Confirmed specificity

- Broad detection range

- Results in less than 3 hours

See for yourself:

www.atcc.org/MDK1

A

[Coumermycin] (ng/mL)
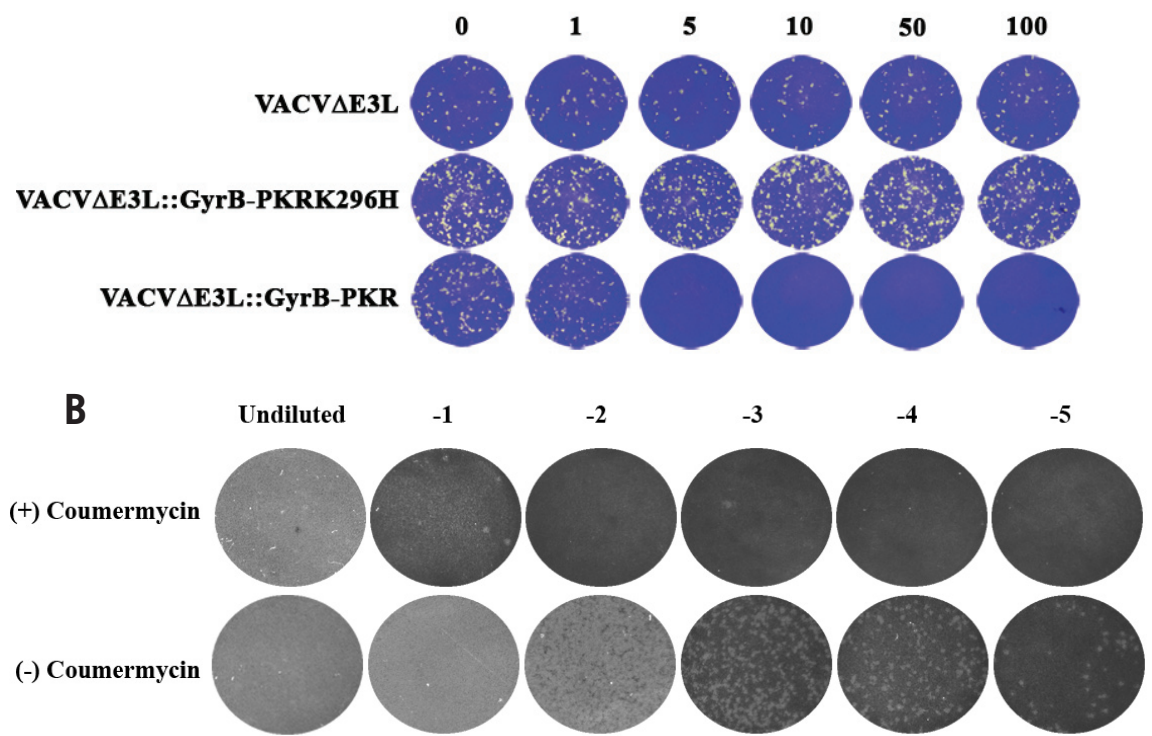

Figure 2. VACV expressing GyrB-PKR is sensitive to the effects of coumermycin. (A) Coumermycin sensitivity of VACV $\triangle E 3 L:: G y r B-P K R$. RK13 cells were pretreated with increasing doses of coumermycin A1 for $16 \mathrm{~h}$ and then infected with 50-100 pfu indicated viruses. At $48 \mathrm{~h}$ post-infection, cells were stained with crystal violet and the plaques quantitated. (B) Coumermycin sensitivity of VACV $J 2 R:: G y r B-P K R$. RK13 cells were mock-treated or treated with $100 \mathrm{ng} / \mathrm{mL}$ coumermycin $\mathrm{A} 1$ for $24 \mathrm{~h}$. The cells were infected with 10-fold serial dilutions of VACV $\triangle \mathrm{J} 2 \mathrm{R}$ ::GyrB-PKR. At $48 \mathrm{~h}$ post-infection, cells were stained with crystal violet and the number of plaques quantitated.

GyrB-PKR was inserted into VACVAE3L by transient dominant selection for mycophenolic acid resistance. Since the kinase activity of this PKR fusion protein is dependent on interaction with coumermycin (9), this virus would be expected to be coumermycin-sensitive $\left(\mathrm{cmr}^{\mathrm{s}}\right)$. In addition to the coumermycin-sensitive virus, a PKR catalytically inactive mutant, VACVAE3L::GyrBPKRK296H, was constructed, which was unable to phosphorylate eIF $2 \alpha$. To confirm that the viruses were able to express the chimeric proteins, RK13 cells were infected with VACV $\triangle \mathrm{E} 3 \mathrm{~L}:: \mathrm{GyrB}-\mathrm{PKR}$, VACVAE3L::GyrB-PKR K296H, and VACV $\triangle \mathrm{E} 3 \mathrm{~L}$ and extracts prepared at $6 \mathrm{~h}$ post-infection. Immunoblot analysis using antibodies against PKR illustrate that only VACVAE3L::GyrB-PKR and VACVAE3L::GyrB-PKRK296H viruses express the GyrB-PKR protein (Figure 1B). To determine whether the GyrB-PKR system was able to function in the context of a vaccinia virus infection in cells in culture, the sensitivity of VACVAE3L::GyrB-PKR, VACVAE3L, and VACVAE3L::GyrBPKRK296H to coumermycin was compared. RK13 cells were pretreated with increasing concentrations of coumermycin and infected with 50-100 pfu of each virus (Figure 2A). For VACVAE3L::GyrB-PKR, sensitivity to coumermycin was observed at concentrations $>5 \mathrm{ng} / \mathrm{mL}$. As expected, both
VACV $\triangle \mathrm{E} 3 \mathrm{~L}$ and the catalytically inactive mutant, VACVAE3L::GyrB-PKRK296H, were resistant to all doses of coumermycin, thereby supporting that the observed sensitivity of VACVAE3L::GyrB-PKR to coumermycin was dependent on the function of the chimeric protein, GyrB-PKR. To determine if the coumermycin-sensitive phenotype was not limited to the expression of the protein in the E3L locus, we also tested the sensitivity to coumermycin with VACV expressing the GyrB-PKR protein in the J2R (thymidine kinase) locus. Clearly this recombinant virus was sensitive to coumermycin by $\geq 100,000$ fold when compared with untreated cells (Figure 2B), demonstrating the versatility of this system for selection of recombinant viruses.

It has been established that the treatment of coumermycin results in the dimerization and activation of GyrB-PKR and leads to the subsequent inhibition of translation $(9,15)$. This inhibition occurs by phosphorylation of a key mediator of translation, eIF $2 \alpha$. To determine that the sensitivity of VACVAE3L::GyrB-PKR to coumermycin was the result of eIF $2 \alpha$ phosphorylation, the level of eIF $2 \alpha$ phosphorylation between the virus infections in the presence or absence of coumermycin was determined. RK13 cells treated with or without coumermycin at $100 \mathrm{ng} / \mathrm{mL}$ for $16 \mathrm{~h}$ were infected at an MOI of 5 to ensure all cells were infected. At $6 \mathrm{~h}$ 


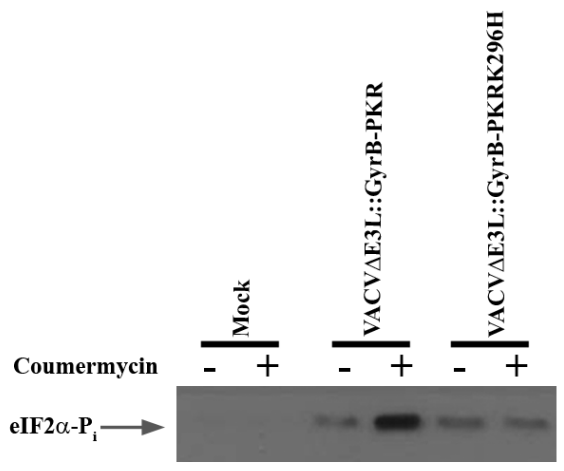

Figure 3. Western blot analysis of elF2a phosphorylation in RK13 cells infected with VACV $\triangle E 3 L:: G y r B-P K R$ in the presence of coumermycin. RK13 cells were pretreated with $100 \mathrm{ng} / \mathrm{mL}$ coumermycin A1 for $16 \mathrm{~h}$ and then infected at an $\mathrm{MOI}$ of 5 with the indicated viruses. At $6 \mathrm{~h}$ post-infection, cell extracts were harvested and subjected to Western blot analysis using antibodies against ser51-phospho-elF2a.

post-infection, extracts were prepared and assayed for eIF $2 \alpha$ phosphorylation. Figure 3 shows that without coumermycin, infection with either VACVAE3L::GyrB-PKR or VACVAE3L::GyrB-PKRK296H led to low levels of eIF $2 \alpha$ phosphorylation compared with mock-infected cells. The low levels of phosphorylation could be attributed to the loss of the E3L gene, which acts to sequester viral dsRNA preventing endogenous PKR activation during a VACV infection $(13,16)$. Comparatively, in the presence of coumermycin, infection with VACVAE3L::GyrB-PKR infection led to an increase in eIF2 $\alpha$ phosphorylation. As expected, infection with the catalytically inactive mutant, VACV $\triangle \mathrm{E} 3 \mathrm{~L}:: \mathrm{GyrB}-$ PKRK296H, did not lead to an increase in eIF2 $\alpha$ phosphorylation following coumermycin treatment. These results suggest that coumermycin mediated dimerization and activation of GyrB-PKR effectively increased eIF2 $\alpha$ phosphorylation and therefore resulted in the sensitivity of VACVAE3L::GyrB-PKR to the antibiotic.

Next, the VACVAE3L::GyrB-PKRsystem was tested for the ability to isolate recombinant viruses. Ideally, the antibiotic should effectively inhibit the replication of viruses that do not contain the desired insert and allow only recombinant viruses to replicate (Figure 4A). pMP $\triangle \mathrm{E} 3 \mathrm{~L}-\mathrm{lacZ}$ was used as the donor plasmid, which upon recombination would place the $\beta$-galactosidase gene in the E3L locus, in place of GyrB-PKR. This would allow for screening and quantitation of the recombinants in the presence of X-gal substrate, resulting in blue plaques. In the absence of coumermycin, the ratio of parental virus to recombinants was $\sim 4000: 1$ $\left(4 \times 10^{6}\right.$ parental to $1 \times 10^{3}$ recombinants), yielding a recombination efficiency of $0.025 \%$ (Figure 4B). Upon treatment with coumermycin, only 10 parental viruses were detected. However, the number of recombinants detected with or without coumermycin treatment was comparable, giving 1000 recombinant plaques without coumermycin to 800 plaques with coumermycin treatment. In the presence of coumermycin, the ratio of parental virus to recombinant virus was $\sim 1: 80$ (10 parental to 800 recombinants). It should be noted that the plaque morphology of the parental virus differed from the recombinant VACV $\triangle \mathrm{E} 3 \mathrm{~L}:: \mathrm{LacZ}$ in the presence of coumermycin, where VACVAE3L::GyrB-PKR plaques appeared as distinct foci in the monolayer, rather than forming clear plaques free of cells. This phenotype only occurred in the presence of the antibiotic. VACVAE3L::LacZ, a wellcharacterized virus with a very limited host range, formed plaques easily distinguishable from VACVAE3L::GyrB-PKR in the presence of coumermycin (data not shown). Individual plaques picked after coumermycin selection routinely yielded only blue recombinant viruses upon replaquing (zero nonblue plaques out of 213 second round plaques assayed). This demonstrates that this selection method can be used to obtain pure virus cultures after minimal rounds of plaque purification. Overall, these results demonstrate that this system is highly efficient in generating recombinant viruses containing genes of interest in a rapid manner. In this example a plasmid containing the gene of interest flanked by homologous arms was used as the donor for homologous recombination. However, since the donor DNA does not need to contain any extraneous sequences, a PCR product containing the gene of interest
A

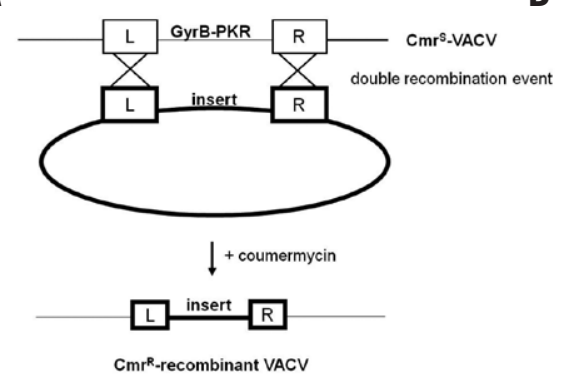

B flanked by homologous arms can be used as the DNA donor using this method.

This study demonstrated that the coumermycin/GyrB-PKR negative selection method is an effective system for the isolation of recombinant VACV viruses. Once a coumermycin-sensitive parental virus was obtained, pure recombinant viruses were rapidly isolated following minimal rounds of plaque purification. The sensitivity to coumermycin was not limited to the GyrB-PKR gene being expressed from the E3L locus, as this phenotype was maintained when GyrB-PKR was expressed from the TK locus (Figure 4), demonstrating the versatility of the system. The GyrB-PKR selection scheme has many advantages over selection methods that result in the rapid isolation of recombinants. Markers such as neomycin (8), hygromycin (17), and puromycin (18), which confer antibiotic resistance, function to enrich the population of recombinants that have taken up the desired DNA through homologous recombination. Enrichment of these recombinants requires multiple rounds of passage and these methods often require the retention of the markers. The use of transient dominant selection markers, such as MPA (1) and GFP (19), requires multiple passages for selection of marker gene uptake followed by resolution for the desired recombinant. In addition, the GyrB-PKR system does not require special cells for the isolation of recombinants unlike thymidine kinase selection $(3,4)$. Furthermore, coumermycin is relatively innocuous to eukaryotic cells, unlike other systems such as MPA and herpes simplex thymidine kinase, which use nucleoside analogs that are potentially mutagenic.

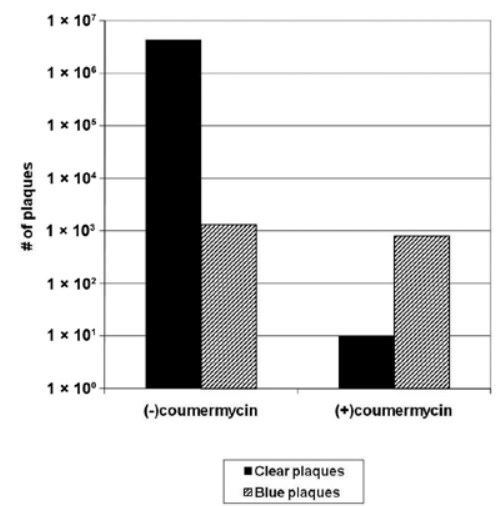

Figure 4. Coumermycin/GyrB-PKR selection of VACV recombinants. (A) Parental $\mathrm{cmr}^{\mathrm{s}}$ VACV expressing GyrB-PKR in the locus of interest undergoes homologous recombination with foreign genetic material flanked by homologous sequences to the locus of interest. Only recombinants that replaced the negative selection marker are resistant (cmrr) to the effects of coumermycin, allowing for their enrichment in the presence of the antibiotic. (B) VACV $\triangle E 3 L$ ::LacZ was generated by in vivo recombination between PMPLacZ and parental virus VACV $\triangle E 3 L:: G y r B-P K R$. Progeny virus was then plaqued out in RK13 cells in the presence or absence of coumermycin. A comparison of the number of recombinants (blue plaques) to parental virus (clear plaques) in the presence or absence of coumermycin was determined. VACVAE3L::GyrB-PKR and recombinant VACV $\triangle E$ EL::LacZ were screened using X-gal and neutral red staining. 


\section{Acknowledgments}

We would like to thank Tom Dever for supplying the vectors pC939 and pC940, which contain GyrB-PKR and GyrBPKRK296H, respectively. This work was funded by the National Institutes of Health (NIH; grant no. AI052347). This paper is subject to the NIH Public Access Policy.

\section{Competing interests}

The authors declare no competing interests.

\section{References}

1. Falkner, F.G. and B. Moss. 1990. Transient dominant selection of recombinant vaccinia viruses. J. Virol. 64:3108-3111.

2. Ball, L.A. 1987. High-frequency homologous recombination in vaccinia virus DNA.J. Virol. 61:1788-1795.

3. Mackett, M., G.L. Smith, and B. Moss. 1984. General method for production and selection of infectious vaccinia virus recombinants expressing foreign genes. J. Virol. 49:857864.

4.Weir, J.P., G. Bajszar, and B. Moss. 1982. Mapping of the vaccinia virus thymidine kinase gene by marker rescue and by cell-free translation of selected mRNA. Proc. Natl. Acad. Sci. USA 79:1210-1214.

5.Holzer, G.W., W. Gritschenberger, J.A. Mayrhofer, V. Wieser, F. Dorner, and F.G.
Falkner. 1998. Dominant host range selection of vaccinia recombinants by rescue of an essential gene. Virology 249:160-166.

6. Perkus, M.E., K. Limbach, and E. Paoletti. 1989. Cloning and expression of foreign genes in vaccinia virus, using a host range selection system. J. Virol. 63:3829-3836.

7. Staib, C., I. Drexler, M. Ohlmann, S. Wintersperger, V. Erfle, and G. Sutter. 2000. Transient host range selection for genetic engineering of modified vaccinia virus Ankara. BioTechniques 28:1137-1148

8. Franke, C.A., C.M. Rice, J.H. Strauss, and D.E. Hruby. 1985. Neomycin resistance as a dominant selectable marker for selection and isolation of vaccinia virus recombinants. Mol. Cell. Biol. 5:1918-1924.

9. Ung, T.L., C. Cao, J. Lu, K. Ozato, and T.E. Dever. 2001. Heterologous dimerization domains functionally substitute for the doublestranded RNA binding domains of the kinase PKR. EMBO J. 20:3728-3737.

10.Ali, J.A., A.P. Jackson, A.J. Howells, and A. Maxwell. 1993. The 43-kilodalton $\mathrm{N}$-terminal fragment of the DNA gyrase B protein hydrolyzes ATP and binds coumarin drugs. Biochemistry 32:2717-2724.

11. Clemens, M.J. 1997. PKR--a protein kinase regulated by double-stranded RNA. Int. J. Biochem. Cell Biol. 29:945-949.

12.Hershey, J.W. 1991. Translational control in mammalian cells. Annu. Rev. Biochem. 60:717-755.

13.Jacobs, B.L., J.O. Langland, and T. Brandt. 1998. Characterization of viral doublestranded RNA-binding proteins. Methods 15:225-232.
14. Kibler, K.V., T.Shors, K.B. Perkins, C.C.Zeman, M.P. Banaszak, J. Biesterfeldt, J.O. Langland, and B.L. Jacobs. 1997. Double-stranded RNA is a trigger for apoptosis in vaccinia virus-infected cells. J. Virol. 71:1992-2003.

15. Friedrich, I., M. Eizenbach, J. Sajman, H. Ben-Bassat, and A. Levitzki. 2005. A cellular screening assay to test the ability of PKR to induce cell death in mammalian cells. Mol. Ther. 12:969-975.

16. Langland, J.O. and B.L. Jacobs. 2004 Inhibition of PKR by vaccinia virus: role of the $\mathrm{N}$ - and C-terminal domains of E3L. Virology 324:419-429.

17.Zhou, J., L. Crawford, X.Y. Sun, and I.H. Frazer. 1991. The hygromycin-resistanceencoding gene as a selection marker for vaccinia virus recombinants. Gene 107:307-312.

18. Sanchez-Puig, J.M. and R. Blasco. 2000. Puromycin resistance (pac) gene as a selectable marker in vaccinia virus. Gene 257:57-65.

19. Wong, Y.C., L.C. Lin, C.R. Melo-Silva, S.A. Smith, and D.C. Tscharke. 2011. Engineering recombinant poxviruses using a compact GFP-blasticidin resistance fusion gene for selection. J. Virol. Methods 171:295-298.

Received 24 August 2010; accepted 11 March 2011.

Address correspondence to Bertram L. Jacobs, CIDV/Biodesign, Box 875401, ASU, Tempe, AZ 85287, USA.e-mail: bjacobs@asu.edu

To order reprints of this article, contact biotechniques@fosterprinting.com
Mesenchymal Stem Cells

Neurons

Endothelial Cells

Fibroblasts

Epithelial Cells

Hepatocytes

Schwann Cells

Melanocytes

Urothelial Cells

Astrocytes

Smooth Muscle Cells

Keratinocytes

DNA/RNA

Cell Transfection

Cell Lysates

Cell Culture Media

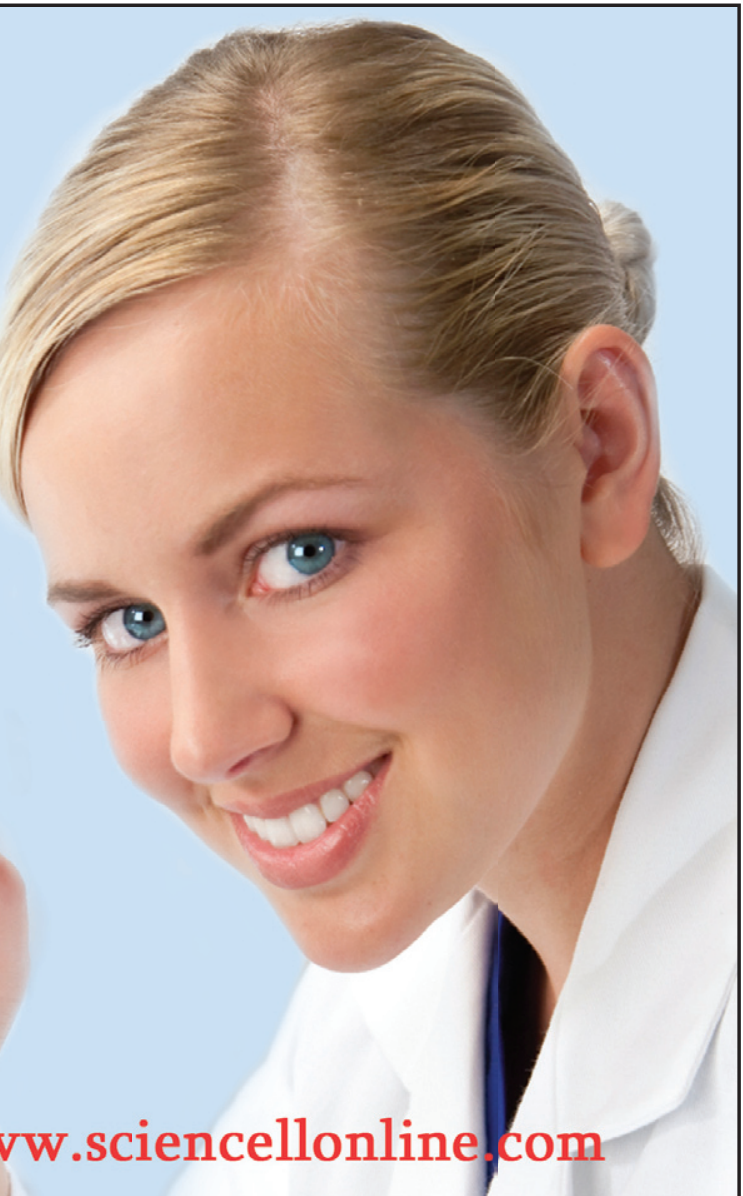

